\title{
Topics
}

\section{Dust Reduction of the Clothing in the International Space Station}

\author{
By Tatsuya AibA ${ }^{1)}$, Go FunABASHi $^{1)}$, Chiemi MatsumurA ${ }^{1)}$, Masaru SATo ${ }^{1)}$, \\ Kazuhito SHIMADA ${ }^{1)}$ and Kenji FUKUTA ${ }^{2)}$ \\ ${ }^{1)}$ Flight Crew Operations and Technology Dept., Japan Aerospace Exploration Agency, Tsukuba, Japan \\ ${ }^{2)}$ Tsukuba Textile Engineering, Tsukuba, Japan
}

(Received June 28th, 2013)

\begin{abstract}
On the ISS, some areas do not satisfy the regulation criteria for acoustic noise. Controlling noise is important not only for protecting the hearing of the astronauts who stay on the ISS but also for the human factor, namely the quality of communication. The causes of noise is derived from atmospheric dust in the ISS. One of the main sources of this dust is the daily inboard clothing worn by the astronauts. The reduction of the dust from the inboard clothing is the most important to control the noise.In general, techniques have been developed to reduce dust when processing materials such as thread and fabric. At this time, we considered a technical process to reduce fuzz on $100 \%$ cotton inboard clothing used on the ISS, and have developed a clothes model and a singeing machine which can burn and remove fuzz on the surface of three dimensional inboard clothing of various shapes. Furthermore, to evaluate process performance, the industrial standard cellophane tape method was used for fuzz occurring on the surface of the processed samples.
\end{abstract}

Key Words: Dust, Reduction, Clothing, ISS

\section{Introduction}

Air-borne dust on the ISS causes problems not only during experiments, but also on the health of the astronauts such as respiratory symptoms.

In addition, noise on the ISS caused by fans, etc., has been noted. This is a serious issue with regards to protecting the hearing of the astronauts and from the human factor, such as the quality of communication. The noise is mainly caused by a clogged filter in the air circulation system by atmospheric dust in the station. Some filters are not accessible unless the air conditioning system is disassembled, so astronauts have to spend many task hours to complete cleaning them.

It is confirmed that, according to measurements by NASA, dust floating in a space shuttle is mainly comprised of fibers from the daily inboard clothing worn by the astronauts ${ }^{1)}$. Reduction of dust originating from inboard clothing is important.

In general, techniques have been developed to reduce dust when processing of materials, such as thread and fabric. ${ }^{2)}$ One example is the singeing process. In this process, fuzz on the surface of fabric comprised of spun yarn, such as cotton, is removed by a gas burner. However, a large production lot is required to meet the processing costs, and the kinds of material which can be used are limited. As a result, it is difficult to apply such dust reduction processing for the inboard clothing of the astronauts because the clothing are mostly commercial off-the-shelf (COTS) products and there are many various sizes and kinds.

In this paper, we considered a fuzz-reducing technique which can be applied to processed products, such as $100 \%$ cotton inboard clothing used on the ISS. Development items include a singeing machine with a control mechanism to singe fuzz on the surface of three dimensional inboard clothing, and a clothes model (mannequin) which can handle various clothing sizes.

\section{Method}

\subsection{Outline of the singeing machine}

To create a luxurious appearance by increasing the silky gloss and texture of a material, a method to burn fuzz and nep of linear and planar raw material surfaces such as thread and cloth by a gas flame during fiber processing has been developed in apparel industry. In this study, we applied this method to three-dimensional clothing (sewn product).

We developed a singeing machine for this study, consisting of a mannequin-shaped clothes model which rotates and a burner which can move forward/backward and up/down, as shown in Fig. 1. The surface of clothing is singed by moving the burner following the shape of contour clothing sample, such as a polo shirt.

Unique mannequin-shaped clothes model was made of wire mesh attached to a $300 \mathrm{~mm} \varphi$ cylinder with flanges on the top and bottom. The diameter of the model changes as the flanges move up or down by adjusting the length of the cylinder. Shirts with chest girth between 800 and $1200 \mathrm{~mm}$ are allowed (Fig. 2).

The burner assembly consists of a gas burner/tank and positioning indicator which measures the distance between the flame and the surface of the clothing. Coupled with rotation of the clothing model, the burner moves up and down, maintaining a specified distance between the flame and the clothing surface. In this way, fuzz on sewn clothing surface can be singed. 


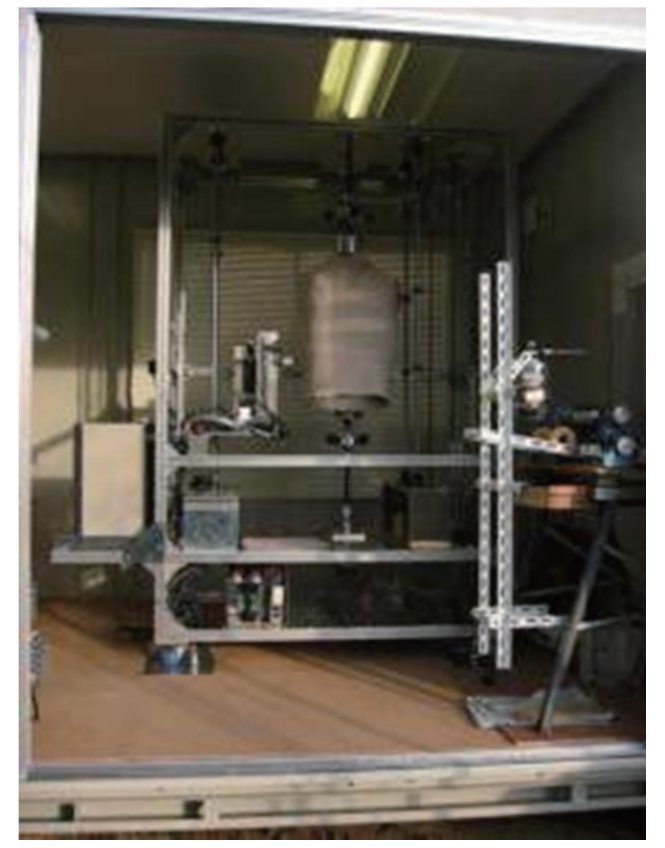

Fig. 1. Singeing machine.



Fig. 2. Continuous sizing of the prototype clothes model.

\subsection{Evaluation of singeing}

We evaluated the effect of singeing process on five $100 \%$ cotton samples (KANOKO fabric), with the distance of $50 \mathrm{~mm}$, clothes peripheral rotation speed of $40 \mathrm{~m} / \mathrm{min}$ and four flame temperatures $\left(855^{\circ} \mathrm{C}, 1,003^{\circ} \mathrm{C}, 1,162^{\circ} \mathrm{C}\right.$, and $\left.1,200^{\circ} \mathrm{C}\right)$.

One method to evaluate the quality of the singeing process was to collect fuzz from the surface of the sample shirts and submit to image analysis. The software was programed to comply with the cellophane tape method by the Japan Textile Products Quality and Technology Center (QTEC). ${ }^{3)}$

To collect fuzz, cellophane tape with a peel force of $3.430 \pm 0.245 \mathrm{~N}$ ( $350 \pm 25 \mathrm{gf}$ ) according to JIS L 1089 testing method was affixed to a sample fabric, and a pressure of $3.92 \mathrm{kPa}(40 \mathrm{gfN} / \mathrm{cm} 2)$ was applied for five seconds, then the tape was peeled off. This procedure was repeated five times. The fuzz attached to the surface of the cellophane tape was photographed by a video microscope (SKM-Z300-PC, Saito
Kogaku Co., Ltd.). The image was analyzed by a software (Image-Pro, Nippon Roper, Inc.), to yield total fuzz fiber length.

Values are expressed as the mean \pm standard deviation. To analyze differences among multiple groups, we used one-way analysis of variance (ANOVA).

\section{Results and Discussion}

Example surface images, before and after the singeing is shown in Fig. 3. The fuzz seen on the surface of the unprocessed product is burned off by the singeing process.

We applied the singeing process on $100 \%$ cotton polo shirts with four flame temperature conditions, and measured the total length of the fuzz collected by the cellophane tape method. The best results were obtained as total fiber length of $62.3 \pm 11.8 \mathrm{~mm}$ at $1,003^{\circ} \mathrm{C}$, and $61.5 \pm 5.3 \mathrm{~mm}$ at $1,162^{\circ} \mathrm{C}$ (Fig. 4).

In this paper, we evaluated four flame temperatures. Flame temperature is one of parameters that should be chosen for singeing process. In near future, we plan to evaluate other singeing processing conditions, which includes distance between the burner and the sample surface, rotation speed of the clothes, and numbers of process repetition.

The standard cellophane tape method used in this paper forcibly peel off fibers. Its mechanism is different from how dust is formed from fuzz of clothes on the ISS. A new method which better simulates actual ISS environment is needed to evaluate the performance of singeing.

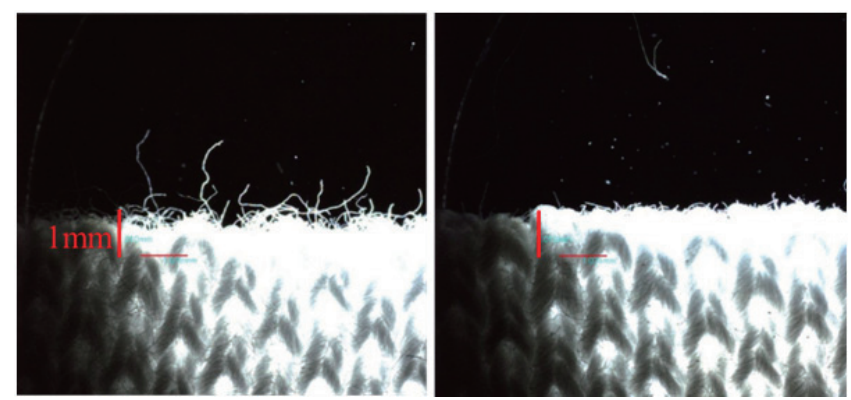

Fig. 3. Image of the sample surface, before and after the singeing process (left: before, right: after).

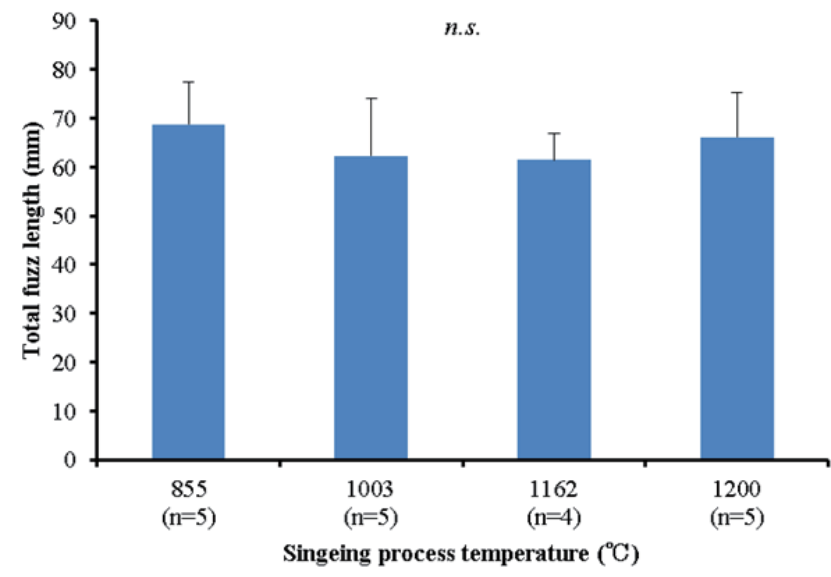

Fig. 4. Total fuzz length. 


\section{Summary}

We considered processing techniques to reduce fuzz from three dimensional clothing on the ISS, and developed a system which can singe fuzz of clothing products. The measurement results of amount of fuzz of cotton shirts suggest that reducing dust in the ISS atmosphere is possible with our singeing method.

\section{References}

1) Goodman, R. J. and Villarreal, L. J.: Space Shuttle Crew Compartment Debris/Contamination., SAE Tech Pap Ser. Soc Automot Eng, (1992) pp.1-10.

2) Collier, A. M.: A Handbook of Textiles, Pergamon Press, Oxford, (1970) pp. 155.

3) Kimura, C. Iwasaki, K. and Takeuchi, Y.: Development of Testing for the Evaluation of Defuzzing (Method of Pressure Sensitive Adhesive Cellophane Tapes), Report of Tokyo Metropolitan Textile Research Institute, 47 (1999) pp.19-25. 